\title{
LITERACIA PARA A SAÚDE EM ALUNOS DO ENSINO SECUNDÁRIO: Relação com a Participação na Saúde Escolar
}

\author{
Paula Maria Dias da Silva ${ }^{1}$ \\ Luís Ângelo Saboga Nunes ${ }^{2}$ \\ Amâncio António de Sousa Carvalho ${ }^{3}$
}

\begin{abstract}
RESUMO
Objetivo: i) caraterizar, em termos sociodemográficos, os alunos da amostra; ii) analisar a relação entre o nível de Literacia para a Saúde (LS) e a participação dos alunos nas atividades de Saúde Escolar (SE). Materiais e métodos: estudo descritivo-correlacional, transversal, de abordagem quantitativa, no qual participaram 499 alunos do ensino secundário público. Foi aplicado um questionário de autopreenchimento que incluía a escala HLS-EU-PT destinada a avaliar o nível de LS. No tratamento de dados utilizamos o SPSS. Resultados: a maioria dos alunos era do sexo feminino (58.3\%) e enquadrava-se no grupo etário dos 16 aos 17 anos (64,5\%). Participaram nas atividades de SE 39,1\% da amostra, dos quais o maior grupo nos âmbitos da educação sexual (23\%) e alimentação saudável (12,6\%). O maior grupo enquadrou-se na LS Suficiente (39,1\%). A categoria de LS designada Alfabetização Limitada para a Saúde atingiu mais de $50 \%$ em todos os domínios, exceto no domínio Promoção da Saúde. O índice de LS Geral não difere significativamente quanto à participação nos programas de SE. Conclusão: Os alunos da amostra apresentam um baixo nível de LS, inferior ao dos participantes em outros estudos. A participação dos alunos da amostra nos programas de SE é baixa, e estas atividades parecem não estar relacionadas com o nível de LS. A equipe de SE deverá estender as suas atividades a mais alunos desta população, procurando aumentar a sua eficácia para elevar o nível de LS.
\end{abstract}

Palavras-chave: Saúde pública. Saúde escolar. Estudantes. Ensinos Fundamental e Médio. Diagnóstico de situação de saúde.

\section{HEALTH LITERACY IN STUDENTS IN HIGH EDUCATION: RELATIONSHIP WITH PARTICIPATION IN SCHOOL HEALTH}

\section{ABSTRACT}

Objective: i) To characterize in the sociodemographic terms the students of the sample; ii) Analyze the relationship between the level of Health Literacy (HL) and the participation of students in School Health (SH) activities. Materials and methods: A descriptive-correlative cross-sectional study with a quantitative approach in which 499 students participated in public high education. A self-completion questionnaire was applied, which included the HLS-EU-PT scale to assess the HL level. In the data treatment we used SPSS. Results: The majority of the students were females (58.3\%) and were in the 16 to 17 age group (64.5\%). SH activities comprised $39.1 \%$ of the sample, of which the largest group in sex education (23\%) and healthy eating (12.6\%). The largest group was enrolled in the HL Sufficient (39.1\%). The HL category called Limited literacy for health reached more than $50 \%$ in all domains, except in the domain of Health Promotion. The General HL index does not differ significantly regarding participation in the SH programs. Conclusion: The students in the sample have a lower HL level, lower than the participants in other studies. The sample students' participation in the SH programs is low, and these activities do not seem to be related to the level of HL. The SH team should extend its activities to more students of this population, seeking to increase its effectiveness to raise the level of LS.

Keywords: Public health. School health. Students. Elementary and high school. Diagnosis of health situation.

\footnotetext{
${ }^{1}$ Licenciatura em Enfermagem. Mestrado em Enfermagem Comunitária. Enfermeira no Centro Hospitalar de Trás-os-Montes e Alto Douro, Unidade de Vila Real, Portugal. conserval.pauladias@gmail.com

2 Universidade Nova de Lisboa, Escola Nacional de Saúde Pública, Lisboa, Portugal.

${ }^{3}$ Universidade de Trás-os-Montes e Alto Douro (Utad), Escola Superior de Saúde, Vila Real Portugal. Centro de Investigação em Estudos da Criança - Ciec-, Universidade do Minho, Braga, Portugal. amancioc@utad.pt
} 
A adolescência é uma etapa do ciclo de vida do ser humano, situada entre os 10 e os 19 anos de idade, sendo caraterizada por mudanças em âmbito fisiológico, psicológico e social, com vistas a uma maior autonomia e à formação da identidade pessoal (UNICEF, 2011).

A procura de novas experiências pelos adolescentes, associada a um certo egocentrismo e a um grande sentimento de invulnerabilidade, levam-nos a adotar comportamentos de risco, muitas vezes com graves prejuízos para a saúde (FONSECA, 2005).

Assim, perante estas caraterísticas é fundamental que este grupo etário possa possuir uma boa literacia para a saúde (LS), que lhe permita tomar as melhores decisões que protejam e promovam a sua saúde e previnam a doença.

A LS foi um conceito introduzido na década de 70, mas que adquiriu uma importância crescente na saúde pública e na saúde com o passar do tempo. Preocupa-se com a capacidade de as pessoas darem uma resposta assertiva às exigências cada vez mais complexas de saúde, numa sociedade moderna e em constante transformação. Deter LS significa colocar a própria saúde e a da sua família e da comunidade em contexto, compreender quais os fatores que a influenciam e saber como lidar com eles. Isso implica que o indivíduo compreenda a informação dada pelos profissionais de saúde relativamente a condições de saúde e opções de tratamento, e conheça a quem recorrer caso necessite de algum auxílio relacionado com o seu estado de saúde (MANCUSO, 2009). Um indivíduo com um nível adequado de LS tem a capacidade de assumir a responsabilidade pela sua própria saúde, pela saúde da sua família e da comunidade (SØRENSEN et al., 2012).

O conceito de LS tem assumido, nos últimos anos, um crescente interesse na relação do indivíduo com o sistema de saúde. A importância desta temática começou a desenhar-se quando se constatou que os níveis de literacia condicionam a forma como as pessoas são ou não capazes de tomar decisões corretas sobre a sua saúde, afetando a qualidade de vida dos indivíduos bem como a daqueles que deles dependem (crianças e idosos, por exemplo). A sua importância, contudo, não se resume ao plano individual, mas tem também implicações nos custos para os sistemas de saúde e para a sua organização (ESPANHA; ÁVILA, 2016).

A Organização Mundial de Saúde define LS como o conjunto de competências cognitivas e sociais que determinam a motivação e a capacidade dos indivíduos para aceder, compreender e usar informação, de forma a promover e manter um bom estado de saúde. Implica a aquisição de conhecimentos, competências pessoais e confiança para agir de forma saudável mediante mudanças de estilo e condições de vida (WHO, 1998).

No entendimento de Saboga-Nunes (2014), a LS pode ser definida como "a conscientização da pessoa aprendente e atuante no desenvolvimento das suas capacidades de compreensão, gestão e investimento, favoráveis à promoção da saúde" (p. 95).

O conceito de LS traduz-se na capacidade de as pessoas tomarem decisões em saúde fundamentadas no decurso da vida em diversos contextos, como em casa, na comunidade, no local de trabalho, no mercado, na utilização do sistema de saúde e no contexto político, possibilitando o aumento do controle pessoal sobre a saúde, a capacidade para procurar informações e para assumir responsabilidades relacionadas com o estado de saúde do próprio indivíduo. 
Estas competências incluem: i) competências básicas em saúde que facilitam a adoção de comportamentos protetores da saúde e de prevenção da doença, bem como de autocuidado; ii) competências do usuário para se orientar no sistema de saúde e agir como um parceiro ativo dos profissionais; iii) competências como consumidor para tomar decisões de saúde na seleção de bens e serviços e agir de acordo com os direitos dos consumidores caso necessário; iv) competências como cidadão, mantendo-se informado, com conhecimento dos seus direitos em saúde, participação no debate de assuntos de saúde e pertença a organizações de saúde e de doentes (WHO, 1998).

No atual clima de transformação dos sistemas sociais, em concreto o sistema de saúde, a integração dos cuidados e a LS são dois conceitos essenciais. O sistema de saúde tem de se adaptar e dar resposta a uma população cada vez mais envelhecida, com mais doenças crônicas e multipatologias, mas também com cada vez maior nível de informação e exigência. Por sua vez, a LS, a capacitação e as tomadas de decisão informadas têm um papel fundamental na definição do percurso do cidadão no Serviço Nacional de Saúde (SNS) (APDH, 2017).

Na concepção de Antunes (2014), as pessoas com baixa LS "apresentam uma menor capacidade de compreensão de conteúdos de material informativo sobre alimentos, doenças crónicas ou sobre o uso de medicamentos, (...), bem como maior dificuldade em pesquisar, selecionar, ler e assimilar a informação em saúde disponível na Internet" ( $\mathrm{p}$. 123).

Na opinião da WHO (2013), a LS limitada está associada a uma menor participação no processo de promoção da saúde e prevenção da doença dos indivíduos. Por sua vez, um nível inadequado de LS está relacionado com atividades de rastreio tardias, opções de saúde de maior risco (como maiores taxas de consumo de tabaco), mais acidentes de trabalho, má gestão de doenças crônicas (como diabetes, asma e infeção pelo HIV), baixa adesão à medicação, erros de medicação, erros de diagnóstico pela má comunicação entre prestadores e os doentes, aumento das taxas de internamento e reinternamento, crescimento da morbilidade e mortalidade prematuras.

A investigação acerca deste objeto é pertinente e atual, pois cada vez há um aumento maior dos custos com os cuidados de saúde, e a LS é "reconhecida como uma questão-chave para o sucesso na reforma dos cuidados de saúde" (ANTUNES, 2014).

Saboga-Nunes e Sørensen (2013) destacam também que a LS "tem um impacto significativo na qualidade de vida e bem-estar dos indivíduos quando as sociedades atravessam condições adversas" (p. 5), sendo um dos recursos que incrementa a resistência e o bem-estar individuais.

A United States Nacional Academy em 1998 estimou que os custos dos cuidados de saúde adicionais, causados pelas limitações da LS, foram cerca de 73 bilhões de dólares. Por sua vez, no Canadá, em 2009, a LS limitada custou mais de 8 bilhões de dólares (WHO, 2013).

Já a Europa gasta milhões de euros em cuidados de saúde, estimando-se que os custos de baixa LS podem ser responsáveis por 3\% a $5 \%$ dos custos totais de saúde no sistema de saúde (EICHLER; WIESER; BRUEGGER, 2009). 
Segundo Antunes (2014), a baixa LS está também associada a taxas de hospitalização mais elevadas e mais prolongadas no tempo, e à ineficácia em lidar com situações de emergência, o que acaba por afetar a relação médico-doente.

Relacionando a LS com os custos do setor, acredita-se que a promoção da mesma, ao mudar o comportamento das pessoas e o seu perfil de utilização do sistema e dos recursos de saúde, pode melhorar a saúde da população e reduzir custos em saúde (WHO, 2013).

De igual modo, a WHO (2013) reconhece os benefícios da junção cidadão e empoderamento, referindo que indivíduos com maior nível de LS participam mais ativamente na prosperidade econômica, têm maior salário e melhor emprego, são mais educados e informados, contribuem mais para atividades comunitárias e desfrutam de uma melhor saúde e bem-estar.

Um estudo realizado entre 2011 e 2013, em Portugal Continental, com uma amostra de 1.180 sujeitos com mais de 15 anos, constatou que 59,9\% dos inquiridos apresentava LS limitada, tendo o nível LS problemática superado ligeiramente o nível LS suficiente. Observou também que os cidadãos portugueses estudados tinham dificuldades consideráveis no que diz respeito aos níveis de processamento da informação, capacidade de acesso, compreensão, capacidade de interpretação e avaliação da informação e a sua utilização em situações diversas e ao longo do seu ciclo vital (COSTA; SABOGA-NUNES; COSTA, 2016).

Em Portugal o governo criou o Programa Nacional para a Saúde, Literacia e Autocuidados, por meio do Despacho no 3.618-A/2016, de 10 de março, para responder aos baixos níveis de LS em Portugal. Também o Programa Nacional de Saúde Escolar (PNSE), indo ao encontro dos objetivos e estratégias do Plano Nacional de Saúde (PNS) (revisão e extensão 2020), pretende obter maiores ganhos em saúde, "através da promoção de contextos escolares favoráveis à adoção de estilos de vida mais saudáveis e à melhoria do nível de literacia para a saúde da comunidade educativa" (DGS, 2015).

É, pois, importante efetivar e aperfeiçoar a literacia na área da saúde. Para isso, é necessário melhorar os processos de comunicação, criando, ajustando e avaliando os processos e suportes de comunicação para ajudar na compreensão das pessoas a quem são dirigidas as atividades (ALMEIDA, 2014).

A Promoção da Saúde em meio escolar, assente nos princípios das Escolas Promotoras de Saúde e no método de trabalho por projeto, deve ter como ponto de partida as necessidades reais da população escolar, desenvolvendo processos de ensino-aprendizagem que possam melhorar os resultados acadêmicos e contribuir para elevar o nível de literacia para a saúde e melhora do estilo de vida da comunidade educativa (DGS, 2015).

Neste sentido, a Saúde Escolar (SE) tem de se apoiar na evidência científica atual. No futuro, no entanto, se quiser aumentar o nível de LS, a responsabilização com a saúde individual e coletiva e o número de anos de vida saudável dos cidadãos, deverá produzir e disseminar a sua própria investigação científica (DGS, 2015). 
É no âmbito desta problemática que realizamos este estudo para o qual delineamos os seguintes objetivos: i) caraterizar em termos sociodemográficos os alunos da amostra; ii) analisar a relação entre o nível de LS e a participação dos alunos nos programas de SE.

\section{MÉTODO}

Trata-se de um estudo observacional, descritivo-correlacional, transversal e de abordagem quantitativa (FORTIN; CÔTÉ; FILION, 2009).

A nossa população-alvo era constituída pelos alunos que frequentavam o ensino secundário de duas escolas secundárias públicas situadas no Concelho de Vila Real, no ano letivo de 2015/2016. Como critérios de inclusão estabelecemos: i) ter idade compreendida entre 14 e 20 anos e ii) frequentar o ensino secundário do ensino público, numa escola da área de abrangência da UCC Vila Real I. A população incluiu 1.237 alunos do ensino secundário (10ำ, 11ㅇ e 12ำ ano), dos quais 646 frequentavam a Escola Secundária A e 591 a Escola Secundária B.

Os critérios de exclusão definidos foram: i) não ter entregado o consentimento dos encarregados de educação para participar no estudo, no caso de jovens com idade inferior a 18 anos; ii) não ter respondido a, pelo menos, $80 \%$ dos itens correspondentes a cada domínio da escala de LS; iii) não estar presente no momento de recolha de dados. A nossa amostra ficou constituída por 499 casos considerados válidos, que obedeciam aos critérios de exclusão, o correspondente a 40,34\% da população-alvo, distribuídos pelas duas escolas: Escola A (321 alunos - 25,95\%) e Escola B (178 alunos - 14,39\%). É, por isso, uma amostragem não probabilística, de conveniência.

Para a recolha de dados optamos por um questionário de autopreenchimento, que se encontrava organizado em quatro partes: i) dados sociodemográficos; ii) Escala de LS; iii) percepção de saúde e utilização de serviços de saúde; iv) conhecimento e participação nos programas/projetos de saúde escolar. Este artigo não abrange a terceira parte.

O nível de LS foi avaliado pela escala HLS-EU-PT (SABOGA-NUNES; S $\varnothing$ RENSEN, 2013), depois da respetiva autorização para a sua utilização. É um instrumento que visa a medir o nível de LS da população. Inclui 47 itens para três domínios de saúde relevantes: cuidados de saúde (16 itens), prevenção de doenças (16 itens) e promoção da saúde (15 itens), ao lado de um índice geral de saúde, compreendendo todos os 47 itens. Estes 3 domínios são avaliados em 4 níveis de processamento de informação (aceder, compreender, avaliar, utilizar) relacionados com a tomada de decisão informada que lhes permita manter uma boa condição de saúde, prevenir doenças e procurar tratamento adequado em caso de doença, resultando numa matriz contendo 12 subdimensões.

Foi atribuído 1 ponto por resposta "muito difícil", 2 pontos por resposta "difícil", 3 pontos por resposta "fácil" e 4 pontos por resposta "muito fácil". Para calcular o nível de literacia, os subníveis foram estandardizados numa escala entre 0 e 50 (melhor possibilidade de LS), com a seguinte fórmula: Índice $=($ média -1$) *(50 / 3)$. O valor 0 representa o mínimo de alfabetização de saúde possível, e o 50 representa a melhor possível. 
Para as quatro dimensões de saúde (geral, cuidados de saúde, prevenção de doenças, promoção da saúde) os valores-limite foram estabelecidos, dividindo os escores em quatro categorias de nível de LS: "Inadequado", "Problemático", "Suficiente" e "Excelente". No total da soma da pontuação foram utilizados como pontos de corte: i) 0-25 pontos - Literacia inadequada; ii) $>25-33$ pontos - Literacia problemática; iii) $>33-42$ pontos - Literacia suficiente; iv) $>42-50$ pontos - Literacia excelente. Para detectar grupos vulneráveis, os níveis "inadequado" e "problemático" foram agrupados num único nível, chamado de "alfabetização limitada para a saúde" (0-33 pontos).

No sentido de respeitar as questões éticas inerentes a este tipo de estudos e de forma a possibilitar a recolha de dados, solicitamos autorização à Direção-Geral de Inovação e Desenvolvimento Curricular por intermédio do Departamento de Monitorização de Inquéritos em Meio Escolar, do qual obtivemos parecer favorável com o no de registo 0480200001 de 08-06-2015. Depois de obtida esta autorização, foi feito o contato e pedido de autorização à direção das respetivas escolas via e-mail, que também deferiram.

Posteriormente foi realizada uma reunião com o(a) diretor(a) de cada uma das escolas envolvidas, de forma a serem explicitados os objetivos da investigação, qual a metodologia pretendida e acordada a forma de recolha de dados. Em seguida foram entregues exemplares do questionário em formato de papel aos diretores das escolas envolvidas, juntamente com o consentimento informado para os encarregados de educação, os quais se responsabilizaram por os entregar aos diretores de turma. Na sequência aqueles entregaram os questionários aos diretores de turma, com a indicação de serem preenchidos em contexto de sala de aula durante o horário letivo dos alunos, de forma a reduzir enviesamentos e garantir a resposta dos estudantes. Foram fornecidas as instruções necessárias para o seu correto preenchimento (leitura de instruções aos alunos, informação de que a resposta era voluntária, confidencial e anônima e foco na importância de preencherem o questionário individualmente). Os diretores de turma fizeram a aplicação dos questionários em sala de aula, recolheram os mesmos em envelope e devolveram aos diretores das escolas, que nos remeteram os mesmos.

A coleta de dados decorreu entre 2 de novembro e 17 de dezembro de 2015, tendo obedecido aos princípios éticos da convenção de Helsínquia, assegurando o consentimento informado dos encarregados de educação, bem como a garantia de confidencialidade e anonimato dos participantes.

Para o tratamento e análise dos dados utilizamos o software informático Statistical Package for the Social Sciences (SPSS, versão 22.0), no qual os dados foram introduzidos. Recorremos à estatística descritiva e inferencial. Em termos de estatística descritiva procedemos ao cálculo das frequências absolutas e relativas para todas as variáveis e, no caso das variáveis de nível de medição rácio, calculamos a média, mediana, desvio padrão, mínimo e máximo. No que respeita à estatística inferencial para testar a hipótese subjacente, recorremos ao teste não paramétrico de Mann-Whitney, uma vez que as variáveis em causa não cumpriam os pressupostos para a utilização dos testes paramétricos. Foi considerado como nível de significância estatística um $p \leq 0.05$ para todas as análises efetuadas (MARÔCO, 2014). 


\section{RESULTADOS}

Do total da amostra ( $n=499$ alunos), a maioria era do sexo feminino (58,3\%), enquadrava-se no grupo etário dos $16-17$ anos (64,5\%) e residia, predominantemente, na área urbana $(72,7 \%)$ e o maior grupo frequentava o 11 응 $(41,1 \%)$ (Tabela 1$)$.

Tabela 1 - Caracterização Sociodemográfica

\begin{tabular}{|l|c|c|}
\hline \multicolumn{1}{|c|}{ Variáveis } & $\mathrm{n}$ & $\%$ \\
\hline Sexo & 208 & 41,7 \\
Masculino & 291 & $\mathbf{5 8 , 3}$ \\
Feminino & & \\
\hline Grupo etário & 119 & 23,8 \\
$14-15$ anos & 322 & $\mathbf{6 4 , 5}$ \\
$16-17$ anos & 58 & 11,6 \\
$\geq 18$ anos & & \\
\hline Tipologia área de residência & 363 & $\mathbf{7 2 , 7}$ \\
APU' & 38 & 7,6 \\
AMU & 89 & 17,8 \\
APR & & 1,8 \\
Não respondeu & 9 & \\
\hline Ano escolaridade & & 29,3 \\
10 o ano & 146 & $\mathbf{4 1 , 1}$ \\
11 o ano & 205 & 29,7 \\
\hline 12 o ano & 148 & 100 \\
\hline Total & 499 & \\
\hline
\end{tabular}

Legenda: APU - Área Predominantemente Urbana; AMU - Área Mediamente Urbana; APR - Área Predominantemente Rural.

No que se refere à participação dos alunos nos programas de SE, a maioria deles nunca tinha participado em atividades de saúde escolar $(68,1 \%)$, e dos que participaram o maior grupo participou em atividade de Educação Sexual (23,0\%), a grande maioria assinalou nunca ter participado no Programa de Alimentação Saudável em Saúde Escolar (Passe) $(94,4 \%)$ e uma ligeira maioria nunca participou no Programa Regional de Educação Sexual em Saúde Escolar (Presse) (58,5\%) (Tabela 2).

Tabela 2 - Participação nos programas de Saúde Escolar

\begin{tabular}{|l|c|c|}
\hline \multicolumn{1}{|c|}{ Variáveis } & $n$ & $\%$ \\
\hline Participação em atividades de Saúde Escolar & & \\
Não & 340 & $\mathbf{6 8 , 1}$ \\
Sim & 159 & 31,9 \\
\hline
\end{tabular}




\begin{tabular}{|l|c|c|}
\hline Âmbito das atividades de SE em que participaram & & \\
Alimentação saudável & 63 & 12,6 \\
Educação sexual & 115 & $\mathbf{2 3 , 0}$ \\
Prevenção de acidentes & 18 & 3,6 \\
Prevenção e controle do tabagismo & 42 & 8,4 \\
Prevenção e controle do alcoolismo & 51 & 10,2 \\
Prevenção da obesidade & 25 & 5,0 \\
Saúde oral & 21 & 4,2 \\
Suporte Básico de vida & 26 & 5,2 \\
Primeiros socorros & 30 & 6,0 \\
Outra & 6 & 1,2 \\
Não sabe/não responde & 9 & 1,8 \\
\hline Participação no Programa Passe & & $\mathbf{9 4 , 4}$ \\
Não & 471 & 5,6 \\
Sim & 28 & $\mathbf{5 8 , 5}$ \\
\hline Participação no Programa Presse & & 41,5 \\
Não & 292 & 100 \\
Sim & 207 & 499 \\
\hline Total & & \\
\hline
\end{tabular}

Quanto ao nível de LS dos alunos, para além da LS Geral (Q1 a Q47), foram avaliados os três domínios: "Cuidados de Saúde" (Q1 a Q16), "Prevenção da Doença" (Q17 a Q31) e "Promoção da Saúde" (Q32 a Q47). A média da pontuação obtida para cada caso varia entre 31,75 no domínio LS associada à "Prevenção da Doença" e 32,84 no domínio associada à "Promoção da Saúde", médias que ainda se situam na categoria de LS Problemática, embora quase no limiar da LS Suficiente. O valor mínimo mais baixo foi obtido no domínio "Cuidados de Saúde" (Tabela 3).

Tabela 3 - Medidas de tendência central e de dispersão

\begin{tabular}{|l|c|c|c|c|c|c|c|}
\hline \multicolumn{1}{|c|}{ Variável } & $\mathrm{n}$ & $\%$ & Média & Mediana & $\mathrm{Dp}$ & Min. & Máx. \\
\hline LS Geral & 499 & 100 & 32,15 & 32,62 & 7,34 & 7,45 & 50,00 \\
\hline LS/ Cuidados de Saúde & 499 & 100 & 31,82 & 32,29 & 7,64 & 1,04 & 50,00 \\
\hline LS/Prevenção da Doença & 499 & 100 & 31,75 & 32,22 & 8,50 & 3,33 & 50,00 \\
\hline LS/Promoção da Saúde & 499 & 100 & 32,84 & 33,33 & 8,40 & 2,08 & 50,00 \\
\hline
\end{tabular}

Tendo em conta os pontos de corte, o maior grupo de alunos apresentava um nível de LS "Suficiente", com uma percentagem que varia entre $36,7 \%$ na LS associada à "Promoção da Saúde" e 39,7\% na LS associada aos "Cuidados de Saúde", logo seguido pelo nível de LS "Problemática", salientando-se, ainda, as reduzidas percentagens de um nível de LS "Excelente". Agrupando as categorias vulneráveis "Inadequado" e "Problemático" num único nível, chamado de "alfabetização limitada para a saúde" (033 pontos), verificamos que, à exceção da LS associada à "Promoção da Saúde", em 
todos os outros domínios da saúde o nível de LS é "Limitado" em mais de $50 \%$ da nossa amostra, e no domínio da "Promoção da Saúde" também anda muito próximo dos $50 \%$ (Tabela 4).

Tabela 4 - Nível de Literacia para a Saúde

\begin{tabular}{|l|c|c|c|c|c|c|c|c|}
\hline \multirow{2}{*}{ Variável } & \multicolumn{2}{|c|}{ Inadequada } & \multicolumn{2}{c|}{ Problemática } & \multicolumn{2}{c|}{ Suficiente } & \multicolumn{2}{c|}{ Excelente } \\
\cline { 2 - 10 } & $\mathbf{n}$ & $\mathbf{\%}$ & $\mathbf{n}$ & $\mathbf{\%}$ & $\mathbf{n}$ & $\mathbf{\%}$ & $\mathbf{n}$ & $\mathbf{\%}$ \\
\hline LS Geral (47) & 78 & 15,6 & 186 & 37,3 & 195 & $\mathbf{3 9 , 1}$ & 40 & 8,0 \\
\hline $\begin{array}{l}\text { LS/ Cuidados de Saúde } \\
\text { (Q1-Q16) }\end{array}$ & 87 & 17,4 & 176 & 35,3 & 198 & $\mathbf{3 9 , 7}$ & 38 & 7,6 \\
\hline $\begin{array}{l}\text { LS/Prevenção da Doença } \\
\text { (Q17-Q31) }\end{array}$ & 95 & 19,0 & 158 & 31,7 & 184 & $\mathbf{3 6 , 9}$ & 62 & 12,4 \\
\hline $\begin{array}{l}\text { LS/Promoção da Saúde } \\
\text { (Q32-47) }\end{array}$ & 96 & 19,2 & 147 & 29,5 & 183 & $\mathbf{3 6 , 7}$ & 73 & 14,6 \\
\hline
\end{tabular}

Não se constataram diferenças estatísticas significativas entre as médias de ordenação dos alunos da LS Geral, que assinalaram ter participado ou não nas atividades de SE e nos dois programas de SE (Passe e Presse) $(p \geq 0,05)$ (Tabela 5).

Tabela 5 - Relação entre a LS Geral e a participação nos Programas de Saúde Escolar

\begin{tabular}{|l|c|c|c|c|c|}
\hline \multicolumn{1}{|c|}{ Variáveis } & $\mathrm{n}$ & $\begin{array}{c}\text { Média de } \\
\text { Ordenação }\end{array}$ & $\begin{array}{c}\text { Valor teste } \\
\text { (MW) }\end{array}$ & $\mathrm{gl}$ & $\mathrm{p}$ \\
\hline LS Geral *QIV.1 & 340 & 251,59 & 26491,00 & - & 0,703 \\
Não & 159 & 246,61 & & & \\
Sim & 471 & 251,43 & 5920,00 & - & 0,334 \\
\hline LS Geral*QIV.2.1 & 28 & 225,93 & & & \\
Não & 292 & 253,05 & 29330,00 & - & 0,550 \\
Sim & 207 & 245,69 & & \\
\hline LS Geral*QIV.3.1 & 2070 \\
Não & Sim & & & & \\
\hline
\end{tabular}

Legenda: LS: Literacia para a Saúde; QIV.1: Já participaste ou participas em atividades da Saúde Escolar; QIV2.1: Participas ou já participaste neste programa (Passe)?; QIV3.1: Participas ou já participaste neste programa (Presse)? gl: graus de liberdade; p: nível de significância; MW: Mann-Whitney.

\section{DISCUSSÃO}

Os resultados demonstram que existe uma proporção ligeiramente maior de alunos do sexo feminino na nossa amostra (58,3\%), não indo ao encontro dos registros nacionais e do município referentes a alunos matriculados no ensino secundário no ano letivo 2014/2015 (PORDATA, 2015), possivelmente explicado pela natureza da amostra, que poderá ter afetado um pouco a sua representatividade. Por outro lado, apresenta uma distribuição dos participantes idêntica aos estudos de Hls-Eu Consortium (2012), Saboga-Nunes e Sørensen (2013) e Ávila, Espanha e Mendes (2015), que usaram a mesma escala de avaliação da LS. 
Observamos na distribuição etária entre os 14 e 18 anos de idade, que prevalecia a categoria dos 16-17 anos, tal como no estudo de Tomás (2014), embora a idade se situasse entre os 14-20 anos neste estudo.

A maioria dos alunos nunca participou em atividades de saúde escolar, sendo também uma elevada percentagem os que nunca participaram no Passe. Já em relação ao Presse, essa percentagem diminuiu para cerca da metade, havendo, assim, uma maior participação neste programa. Isso poderá ser explicado, por um lado, pela data de início da introdução do Passe (2009), aplicado inicialmente apenas a alunos do 1 o e 2 o ciclos, o que implica que somente os alunos do 10 o ano tivessem sido por ele abrangidos, enquanto o Presse tinha sido aplicado ao ensino secundário desde 2009.

Após análise dos resultados obtidos respeitantes ao nível de LS, verificamos que a maioria dos alunos deste estudo enquadrava-se no nível limitado, tal como em Saboga-Nunes e Sørensen (2013), e em oposição aos resultados dos estudos de HIs-Eu Consortim (2012) e Ávila, Espanha e Mendes (2015), nos quais o nível de LS da maioria dos inquiridos foi adequado (associação dos níveis Suficiente e Excelente). Ressalta-se, no entanto, mais uma vez, que em todos estes estudos - portugueses e europeus -, as amostras diferem da presente pesquisa, uma vez que incluíram toda a população com mais de 15 anos de idade, podendo a explicação para estas diferenças residir neste fato.

Fazendo uma comparação do nível médio de LS entre os alunos desta amostra e os participantes nos estudos referidos anteriormente, verifica-se que, à exceção da LS associada à "Promoção da Saúde", os alunos deste estudo apresentam um nível médio de LS na ordem dos 32\%, o que é ligeiramente inferior ao Hls-Eu Consortium (2012) e Ávila, Espanha e Mendes (2015). No domínio da "Promoção de Saúde", o nível médio de LS no presente estudo (32,8\%) é ligeiramente superior ao europeu $(32,5 \%)$ e ao português $(32,1 \%)$, mas bastante semelhante, rondando os $32 \%$.

Não se observou qualquer relação estatisticamente significativa entre o nível de LS e a participação nos programas de SE, o que não se coaduna com a literatura consultada, em que é exposto que a Educação para a Saúde está fortemente relacionada com o nível de LS (CARVALHO; CARVALHO, 2006).

A principal limitação deste estudo relaciona-se com a natureza da amostra, uma vez que se trata de uma amostra de conveniência e, portanto, não probabilística, podendo não ter permitido uma representatividade muito fiel da população, o que dificulta a generalização das inferências da amostra para a população deste.

\section{CONCLUSÕES}

O perfil dos alunos participantes caracterizou-se por maioritariamente ser do sexo feminino, com idade compreendida entre os 16 e 17 anos, residentes em área predominantemente urbana e frequentarem o $11^{\circ}$ ano de escolaridade.

O nível de LS dos alunos desta amostra é, no geral e em todos os domínios (cuidados de saúde, prevenção da doença e promoção da saúde), limitado na ordem dos $50 \%$, podendo considerar-se que os alunos participantes no estudo possuem, na sua 
maioria, um baixo nível de literacia. Este nível de LS é ainda inferior ao mostrado por participantes em outros estudos realizados em Portugal, embora mais velhos do que os da presente investigação.

Verificamos, ainda, que não existe relação de dependência entre o nível de LS e a participação nos programas de SE desenvolvidos nas escolas que estes alunos frequentam. Este último achado parece indicar que os referidos programas e projetos não abrangeram todos os alunos e terão sido pouco eficazes na promoção da LS.

A necessidade de investigação em LS em adolescentes, sobretudo em Portugal, onde se constata uma escassez de pesquisas sobre a matéria, deixa em aberto a possibilidade da realização de estudos futuros que corroborem ou não os resultados encontrados neste, bem como outros resultados que os complementem, de caráter longitudinal, que possam avaliar o efeito da efetivação dos Programas de SE no nível de LS.

\section{REFERÊNCIAS}

ALMEIDA, C. V. A literacia: uma acessibilidade a priori no verdadeiro caminho para a capacitação. In: CONFERÊNCIA REFLEXÃO, AVALIAÇÃO, 2014, Lisboa. Atas [...]. Lisboa: CRA, 2014. Disponível em: https://www. researchgate.net/. Acesso em: 15 abr. 2018.

ANTUNES, M. L. A literacia em saúde: investimento na promoção da saúde e na racionalização de custos. In: JORNADAS APDIS, 11., As bibliotecas da saúde, que futuro? 2014, Lisboa. Actas [...]. Lisboa: Apdis, 2014. Disponível em: https://repositorio.ipl.pt/handle/10400.21/3582. Acesso em: 15 abr. 2018.

APDH. Associação Portuguesa Para o Desenvolvimento Hospitalar. Participação dos cidadãos e literacia em saúde. In: CONFERÊNCIA INTEGRAÇÃO DE CUIDADOS E LITERACIA EM SAÚDE. CAPACITAR O CIDADÃO NO SNS, 2017, Lisboa. Atas [...]. Lisboa: APDH, 2017. Disponível em: http://www.apdh.pt/en/node/2299. Acesso em: 15 abr. 2018.

ÁVILA, P.; ESPANHA, R.; MENDES, R. Inquérito à literacia em saúde Portugal. Relatório Final. Lisboa: Cies-IUL; Fundação Calouste Gulbenkian, 2015. Disponível em: https://ciencia.iscte-iul.pt/publications/inquerito-a-literacia-em-saude-em-portugal/27146. Acesso em: 26 fev. 2017.

CARVALHO, A. A. S.; CARVALHO, G. S. Educação para a saúde: conceitos, práticas e necessidades de formação. Um estudo sobre as práticas de educação para a saúde dos enfermeiros. Loures: Lusociência, 2006.

COSTA, A.; SABOGA-NUNES, L.; COSTA, L. Avaliação do nível de literacia para a saúde numa amostra portuguesa. Observações Boletim Epidemiológico, Lisboa: Instituto Nacional de Saúde Doutor Ricardo Jorge, v. 17, 2a série, p. 38-40, 2016. Disponível em: http://repositorio.insa.pt/bitstream/10400.18/4111/1/Boletim_Epidemiologico_Observacoes_N17_2016_artigo9.pdf.. Acesso em: 10 mar. 2018.

DGS. Direção-Geral da Saúde. Programa nacional de saúde escolar. Lisboa: DGS, 2015.

EICHLER, K.; WIESER, S.; BRUEGGER, U. The costs of limited health literacy: A systematic review. International Journal of Public Health, Bern, v. 54, n. 5, p. 313-324, July. 2009. Available from: https://www.ncbi. nlm.nih.gov/pubmed/19644651. Cited: 23 jun. 2018.

ESPANHA, R.; ÁVILA, P. Health Literacy Survey Portugal: a Contribution for the Knowledge on Health and Communications. Procedia Computer Science, Amsterdam, v. 100, p. 1.033-1.041, out. 2016. Available from: https://www.researchgate.net/profile/Rita_Espanha/publication/308899529_Health_Literacy_Survey_Portugal_a_Contribution_for_the_Knowledge_on_Health_and_Communications-review_under_responsibility_of_SciKA_-Association_for_Promotion_and_Dissemination_of_Scientific_Knowledge/links/ 57f60fd508ae91deaa5e3e6b.pdf. Cited: 23 jun. 2018.

FORTIN, M-F.; CÔTÉ, J.; FILION, F. Fundamentos e etapas do processo de investigação. Loures: Lusodidacta, 2009.

FONSECA, H. Compreender os adolescentes: um desafio para pais e educadores. 3. ed. Barcarena: Editorial Presença, 2005.

HLS-EU CONSORTIUM. Comparative report of health literacy in eight EU Member States. The European Health Literacy Survey HLS-EU. Maastricht, 2012. 92p. Available from: http://www.health-literacy.eu. Cited: 26 fev. 2017. 
MANCUSO, J. Assessment and measurement of health literacy: An integrative review of the literature. Nursing and Health Sciences, Rockville Pike, v. 11, n.1, p. 77-89, Mar. 2009. Available from: https://www. ncbi.nlm.nih.gov/pubmed/19298313. Cited: 23 jun. 2018.

MARÔCO, J. Análise estatística com o SPSS Statistics. 6. ed. Lisboa: Report Number, 2014.

PORDATA. Base de Dados Portugal Contemporâneo. Municípios, educação, alunos do ensino não superior. Lisboa, 2015. Disponível em: www.pordata.pt/. Acesso em: 26 fev. 2017.

PORTUGAL. Despacho no 3618-A/2016, de 10 de março. Determina a criação do Programa Nacional de Educação para a Saúde, Literacia e Autocuidados. Diário da República, v. 49, Série II, 2016.

SABOGA-NUNES, L. Literacia para a saúde e a conscientização da cidadania positiva. Revista Referência, Coimbra, v. 11, Série 3, p. 94-99, feb. 2014. Suplemento.

SABOGA-NUNES, L.; SØRENSEN, K. The European Health Literacy Survey (HLS-EU) and its Portuguese cultural adaptation and validation (HLS-PT). [abstract]. Atención Primaria, p. 45-46, may. 2013. Paper apresentado no 1st World Congress of Children and Youth Health Behaviours and 4th National Congress on Health Education, p. 23-25, May 2013, Viseu. Available from: https://research.unl.pt/en/publications/ the-european-health-literacy-survey-hlseu-and-its-portuguese-cultural-adaptation-and-validation-hlspt(bdd59a62-2992-49e6-9059-14933114a580)/export.html. Cited: 26 fev. 2017.

SØRENSEN, K. et al. Consortium Health Literacy Project European. Health literacy and public health: A systematic review and integration of definitions and models. BMC Public Health, London, v. 12, n. 80, p. 1-13, jan. 2012. Available from: https://bmcpublichealth.biomedcentral.com/track/pdf/10.1186/14712458-12-80. Cited:

26 fev. 2017.

TOMÁS, C. C. Literacia em saúde na adolescência. 2014. 692 f. Tese (Doutorado em Ciências de Enfermagem) - Instituto de Ciências Biomédicas de Abel Salazar, Universidade do Porto, Porto, 2014.

UNICEF. United Nations Children's Fund. Situação mundial da infância. Adolescência, uma fase de oportunidades. New York: Unicef, 2011. 148 p. Disponível em: https://www.unicef.org/brazil/pt/br_sowcr11web.pdf. Acesso em: 24 maio 2014.

WHO. World Health Organization. Health promotion glossary. Geneva, 1998. 36 p. Available from: http:// www.who.int/healthpromotion/about/HPR\%20Glossary\%201998.pdf. Cited: 29 dez. 2016.

WHO. World Health Organization. Health Literacy. Solid Facts. Copenhagen, 2013. 86 p. Available from: http://www.euro.who.int/en/what-wedo/health-topics/environment-and-health/urbanhealth/publications/2013/health-literacy.-the-solid-facts. Cited: 29 dez. 2016. 